\title{
Endobronchial suction catheter guided nasotracheal intubation with preformed nasotracheal tube
}

\author{
Jia Song, Taehee Pyeon, Daehoon Kim, Changhoon Lee, Joungmin Kim \\ Department of Anesthesiology and Pain Medicine, Chonnam National University Medical School, Gwangju, Korea
}

Received September 16, 2019

Revised September 25, 2019

Accepted September 25, 2019

\section{Corresponding author}

Joungmin Kim

Department of Anesthesiology and

Pain Medicine, Chonnam National

University Medical School,160,

Baekseo-ro, Gwangju 61469, Korea

Tel: +82-62-220-6895

Fax: + 82-62-232-6294

E-mail: tca77@hanmail.net

ORCID:

http://orcid.org/0000-0003-1135-1968

\begin{abstract}
Nasotracheal intubation is commonly used in surgery requiring space of the orofacial field. The endotracheal tube used for nasotracheal intubation is either a conventional tube or a preformed nasotracheal tube considering nasal passage. Conventional nasotracheal intubation starts with blind passage of an endotracheal tube via the nostril followed by laryngoscopy-assisted intubation through the vocal cord with or without the aid of Magill forceps. During intubation, anesthesiologists may experience interruption of endotracheal tube passage because of resistance in nasal cavity. It is known that any excessive force may significantly increase the risk of damage to nasal structures. One way to prevent this damage is Guided-technique. Guided technique is easier, cheaper and safer than conventional technique. Here we report a case of successful endotracheal intubation with preformed nasotracheal tube following endobronchial suction catheter guidance. Guided technique with endobronchial suction catheter is useful for nasotracheal intubation with preformed nasotracheal intubation tube.
\end{abstract}

Keywords: Nasotracheal intubation; Endobronchial suction catheter; Preformed nasotracheal tube; Guided-technique

\section{INTRODUCTION}

Nasotracheal intubation is frequently used to maintain airway in general anesthesia for surgeries of the head and neck region. This method has advantage in that it provides good accessibility to surgical field while minimizing interference due to tube in nasopharynx. Usually, preformed nasotracheal tubes and conventional endotracheal tubes are used for nasotracheal intubation. A preformed nasotracheal tube is pre-bent to prevent kinking of the tube. It is also longer than a conventional orotracheal tube because the tube must pass through the nasal cavity and provide space for fixation.

Anesthesiologists often feel resistance when the tube passes through the nasal cavity during nasotracheal intubation. The anesthesiologist can try switching to the other nasal cavity if the tube feels resistance as it passes through one nasal cavity. If the resistance is felt in both nostrils, it induces anesthesiologists to make decision about ongoing intubation with more force or using another method. In many studies, the more excessive force is added to tube, the more increased risk of damage to nasal structure [1,2]. Many studies have been performed to solve this problem. To prevent interruption of the tube, many preparations have been developed, such as pre-warming of the tube with warm saline solution, choosing the correct nostril for intubation, injecting vasoconstrictor to nasal cavity, and using nasal airway before nasotracheal intubation [3-5]. In addition, a

This is an Open Access article distributed under the terms of the Creative Commons Attribution Non-Commercial License (http://creativecommons.org/licenses/by-nc/4.0) which permits unrestricted noncommercial use, distribution, and reproduction in any medium, provided the original work is properly cited. Copyright $(\subset$ Medical Biological Science and Engineering. 

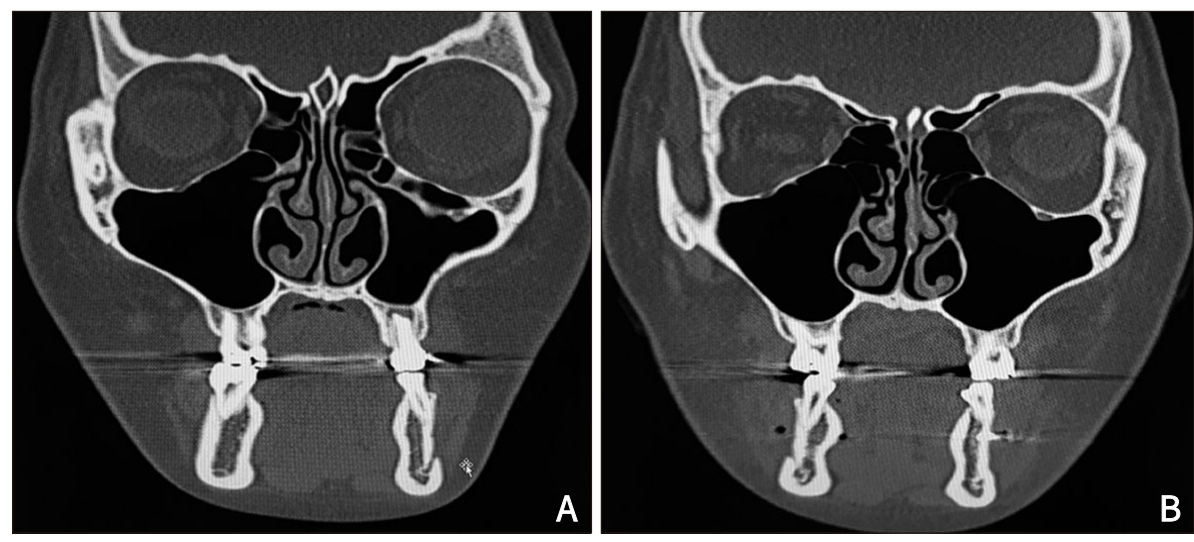

Fig. 1. Facial computed tomography showing no definitive abnormal finding such as cyst, septal deviation, or bony spur. (A) Preoperative, (B) Postoperative.

guided technique using bougie or nasogastric tube has been studied [6,7]. However, these studies used conventional endotracheal tubes.

Here, we report a case of successful intubation with preformed nasotracheal tube using an endobronchial suction catheter as an inducer even in narrow nasal passages. This suggested the endobronchial suction catheter as guidance for endotracheal intubation using preformed nasotracheal tubes.

\section{CASE REPORT}

A 19-year-old Korean woman was planned to undergo mandible bilateral sagittal split ramus osteotomy (BSSRO) because of mandibular prognathism. She had no underlying diseases or abnormal preoperative evaluation results including facial CT (Fig. 1). The operation included intraoral procedure. Thus, we planned nasotracheal intubation with preformed nasotracheal tube. After the patient entered the operating room, we confirmed the patient's name and operation site followed by standard monitoring. Induction of anesthesia was performed with propofol $100 \mathrm{mg}(1.6 \mathrm{mg} / \mathrm{kg})$ and rocuronium $50 \mathrm{mg}(0.8 \mathrm{mg} / \mathrm{kg})$. Spontaneous breathing disappeared, indicating well mask ventilation. In the first intubation attempt, polar preformed nasotracheal tube \#6.5 (Smith medical) (Fig. 2) was tried. However, it failed because of interruption of the tube in both nostrils. The cause of the failed intubation might be due to narrow nostril. Thus, smaller nasotracheal tube (polar preformed nasotracheal tube \#6.0) was used in the next intubation. A preformed nasotracheal tube \#6.0 was the narrowest one that was available to us. If we wanted the next attempt intubation

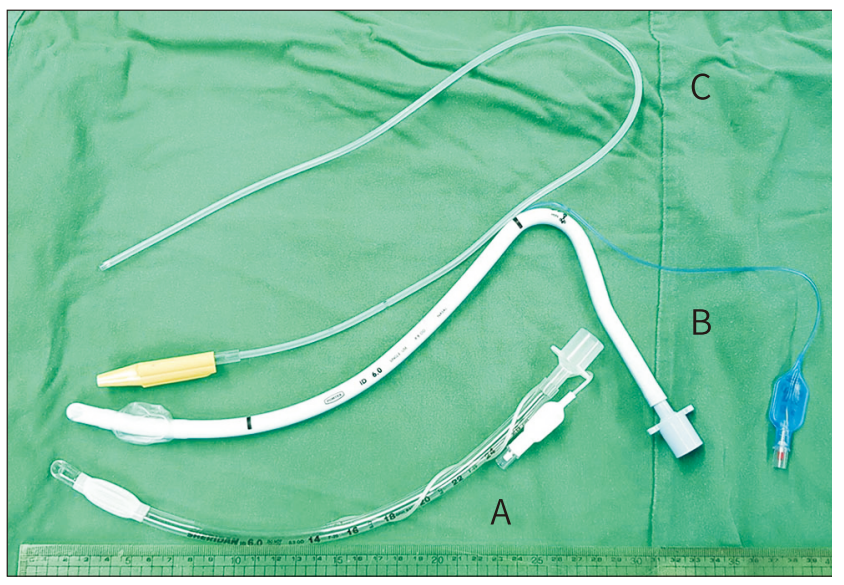

Fig. 2. Tube length comparison. (A) Conventional endotracheal tube, (B) Preformed nasotracheal tube, and (C) Endobronchial suction catheter.

with narrower endotracheal tube, we must use conventional endotracheal tube. Another choice was using equipment for guidance of preformed nasotracheal tube. Some studies have reported guided technique for nasotracheal intubation [6,7]. We inserted the endobronchial suction catheter with color coded connectors [10 $\mathrm{Fr} / \mathrm{Ch}(3.3 \mathrm{~mm})$, Covidien] to cover preformed nasotracheal tube length to the left nostril and confirmed that the catheter tip was inserted to oral cavity by laryngoscopy. Preformed nasotracheal tube couldn't pass the suction catheter because connector of the suction catheter was larger than preformed nasotracheal tube. The connector of the suction catheter was removed to insert preformed nasotracheal tube to nostril. Subsequently, preformed nasotracheal tube \#6 entered gently along the endobronchial suction catheter. During the procedure, the assistant grabbed the tip of suction catheter tightly not to enter to nostril together with preformed nasotracheal tube. In laryngoscopy view, preformed nasotracheal tube entered 

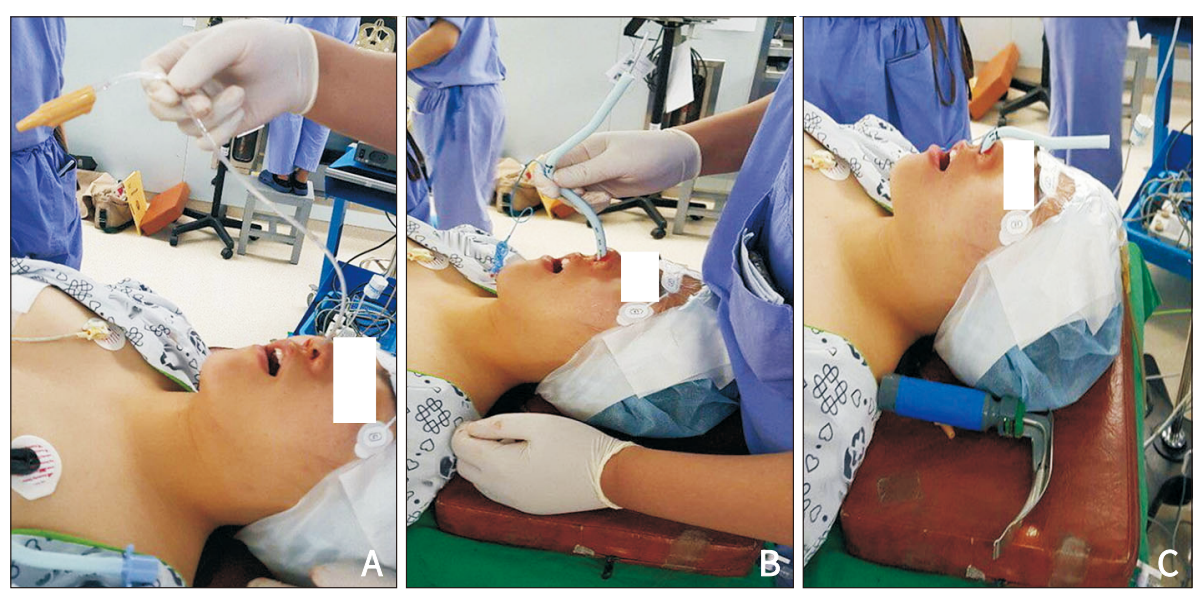

Fig. 3. Intubation was attempted as follows. (A) Inserting the endobronchial suction catheter to the left nostril, (B) Advancing preformed nasotracheal tube with threaded by endobronchial suction catheter, (C) Removing endobronchial suction catheter after successful intubation.

the oral cavity (Fig. 3). We used Magill forceps to induce preformed nasotracheal tube toward vocal cord and completed the intubation. Without problem, nasotracheal intubation was successful. In the procedure, the patient's vital sign was stable. The procedure was finished without any problem. At the time of extubation, there was no epistaxis or nasal pain. She stayed at postanesthetic recovery room without any problem. Then she went to the ward. In the ward, she did not experience any adverse event. She was discharged after 3 days.

\section{DISCUSSION}

This case showed that endobronchial suction catheter could serve as a useful guidance for nasotracheal intubation using preformed nasotracheal tube. Nasotracheal intubation is commonly required in patients undergoing head and neck surgery including intraoral approaches. However, it is more traumatic than orotracheal intubation [3,8]. Because nasal cavity is narrower than oral cavity with fragile structures like turbinate [2]. The upper pathway underneath the middle turbinate seems to be susceptible because of a middle turbinate is a fragile and high vascular structure. Thus, the lower pathway is thought to be a safer passage for endotracheal tube. Trauma of the middle turbinate may result in turbinate fracture, severe epistaxis, cerebrospinal fluid rhinorrhea, and dysfunction of olfactory nerve [8,9]. Since conventional technique includes blind procedure which starts with blind passage of a tracheal tube via the nostril, anesthesiologists have difficulty passing the tube into the lower pathway on purpose [6].
Many anesthesiologists feel a sense of threshold pressure when advancing a tracheal tube to nostril for nasotracheal intubation. Any excessive force may significantly increase the risk of damage to nasal structures. A previous study has noticed that $43 \%$ of nasotracheal intubations are felt with resistance and anesthesiologists have to manipulated the tube more than once [5]. Interruption of the tube during nasotracheal intubation because of resistance might be a distinct and difficult problem to anesthesiologists. Thus, many studies have been performed to solve the problem. To prevent interruption of the tube, many preparations have been developed, including pre-warming of the tube by warm saline solution, choosing the correct nostril for intubation, injecting vasoconstrictor to nasal cavity, and using nasal airway before nasotracheal intubation [3-5].

Another solution is the use of fiberscope. The fiberscope has advantage to secure direct view about nasal cavity. Thus, it can decrease adverse effects of nasotracheal intubation such as epistaxis and make nasotracheal intubation easier by finding out the best nostril in the patient who may experience interruption of tube [10]. However, it takes long training time. In addition, it is expensive.

Other studies have developed methods using Seldinger technique. In these methods, nasotracheal tube passed lower nasal pathway more than upper nasal pathway by guide effect. These methods have advantages in that they are easier and cheaper than fiberoptic nasotracheal intubation.

Bougie [6], nasogastric tube [7] and polyvinyl chloride suction catheter [11] were used for Seldinger technique in previous studies. Guidance with them, less complications such as epistaxis and laceration were occurred, tubes passed the 
lower pathway more than the upper pathway. Also success rate of intubation was higher than conventional intubation.

Since guided technique like Seldinger technique has easier and safer nasal passage than conventional technique, it is valuable and useful for nasotracheal intubation. Special equipment or training course is not needed for guided technique. In our case, the position of the tube was not checked visually, but it could be assumed that the tube passed in lower pathway with guidance effect. We think that our case is a specific one because the use of guided technique with preformed nasotracheal tube is rare. There are few papers about nasotracheal intubation using guided technique with preformed nasotracheal tube. Thus, we believe that this is a useful technique for difficult nasotracheal intubation cases when resistance is felt during nasal passage.

Endobronchial suction catheter is a good option because of the angled and long character of preformed nasotracheal tube. However, further studies are needed to determine the safety and economics of using suction catheter.

\section{CONFLICT OF INTEREST}

No potential conflict of interest relevant to this article was reported.

\section{REFERENCES}

1. Smith JE, Reid AP. Asymptomatic intranasal abnormalities influencing the choice of nostril for nasotracheal intubation.
Br J Anaesth 1999;83:882-6.

2. Ahmed-Nusrath A, Tong JL, Smith JE. Pathways through the nose for nasal intubation: a comparison of three endotracheal tubes. Br J Anaesth 2008;100:269-74.

3. Hall CE, Shutt LE. Nasotracheal intubation for head and neck surgery. Anaesthesia 2003;58:249-56.

4. Mahajan R, Gupta R. Another method to avoid trauma during nasotracheal intubation. Anesth Analg 2005;101:928-9.

5. Prasanna D, Bhat S. Nasotracheal intubation: an overview. J Maxillofac Oral Surg 2014;13:366-72.

6. Abrons RO, Zimmerman MB, El-Hattab YMS. Nasotracheal intubation over a bougie vs. non-bougie intubation: a prospective randomised, controlled trial in older children and adults using videolaryngoscopy. Anaesthesia 2017;72:1491500.

7. Lim CW, Min SW, Kim CS, Chang JE, Park JE, Hwang JY. The use of a nasogastric tube to facilitate nasotracheal intubation: a randomised controlled trial. Anaesthesia 2014;69:5917.

8. St Mont G, Biesler I, Pförtner R, Mohr C, Groeben H. Easy and difficult nasal intubation--a randomised comparison of Macintosh vs Airtraq® laryngoscopes. Anaesthesia 2012;67:1328.

9. Williams AR, Burt N, Warren T. Accidental middle turbinectomy: a complication of nasal intubation. Anesthesiology 1999;90:1782-4.

10. Kwon MA, Song J, Kim S, Ji SM, Bae J. Inspection of the nasopharynx prior to fiberoptic-guided nasotracheal intubation reduces the risk epistaxis. J Clin Anesth 2016;32:7-11.

11. Anwer HMF, Ibrahim AA. Suction catheter guidance of the endotracheal tube to facilitate nasal intubation: a double blind, randomised clinical trial. Middle East J Anaesthesiol 2018;25:155-64. 